\title{
Conocimiento sobre Consentimiento Informado en Docentes, Estudiantes y Pacientes de la Carrera de Odontología
}

\author{
Knowledge on Informed Consent in Teachers, \\ Students and Patients of the Faculty of Dentistry
}

Catalina Loza Rebolledo; Bernarda Salgado Miranda; Jonathan Sánchez

Vergara; Fernanda Sarmiento Ibaceta \& Denisse Lagos Tissie

LOZA, R. C.; SALGADO, M. B.; SÁNCHEZ, V. J.; SARMIENTO, I. F. \& LAGOS, T. D. Conocimiento sobre consentimiento informado en docentes, estudiantes y pacientes de la carrera de odontología. Int. J. Odontostomat., 14(3):424-429, 2020.

RESUMEN: Determinar el nivel de conocimiento respecto al procedimiento de consentimiento informado que presenta una muestra de docentes, estudiantes y pacientes odontológicos. Se aplicó un cuestionario anónimo a 271 estudiantes, 58 docentes y 260 pacientes de una Clínica Odontológica Universitaria. El análisis de datos se realizó aplicando el test chi-cuadrado. Los estudiantes, en su mayoría, presentan un conocimiento alto sobre $\mathrm{Cl}$. Un $60,61 \%$ de los docentes muestran un grado de conocimiento regular y un 39,39 \% presenta conocimiento alto. Los pacientes, en su mayoría, presentan un conocimiento regular $(58,41 \%)$. Es importante aumentar la investigación del tema y la formación continua que introduzca conocimientos sobre autonomía del paciente. De esta manera, crearemos conciencia en los docentes del área y fortaleceremos el conocimiento de las nuevas generaciones, contribuyendo al cumplimiento de nuestro mandato legal y la contribución al ejercicio pleno del derecho a la autonomía de nuestros pacientes.

PALABRAS CLAVE: consentimiento informado, autonomía del paciente, derechos del paciente.

\section{INTRODUCCIÓN}

El consentimiento informado $(\mathrm{Cl})$ es el proceso mediante el cual a un paciente se le proporciona la información suficiente para tomar una decisión razonada acerca del procedimiento propuesto. Es otorgado por el paciente sin ninguna coacción o fraude, basado en su entendimiento razonable de lo que ocurrirá, incluyendo la necesidad de realizar el tratamiento, su beneficio y los riesgos de éste, la existencia de cualquier procedimiento alternativo disponible y los riesgos del rechazo al tratamiento propuesto (Centro Interdisciplinario de Estudios en Bioética, 2006).

En Chile, hasta el año 2012, sólo se exigía Cl ante la aplicación del examen de detección del virus de la inmunodeficiencia humana, para la manifestación en vida de la voluntad de donar órganos y para la internación de pacientes con dificultades mentales (Ministerio de Salud, 2000, 2017, 2019).

La Ley 20.584 (de Derechos y Deberes del paciente), que entró en vigencia el año 2012, empieza a exigir la aplicación del $\mathrm{Cl}$ en todas las prestaciones clínicas, lo cual queda establecido en sus artículos 14 y 15 y es operacionalizado posteriormente en el Decreto 31/2012 del Ministerio de Salud de Chile (Ministerio de Salud, 2012a), estableciendo que "Toda persona tiene derecho a otorgar o denegar su voluntad para someterse a cualquier procedimiento o tratamiento vinculado a su atención de salud (...)" e indicando las directrices para su redacción y aplicación en casos especiales (atenciones no invasivas, atenciones de urgencia vital, investigación, pacientes discapacitados mentalmente (Subsecretaría de Salud Pública, 2019).

El objetivo de este estudio fue determinar el nivel de conocimiento respecto al procedimiento de $\mathrm{Cl}$ que presentan docentes, estudiantes y pacientes que acuden a una Clínica Odontológica Universitaria en la Ciudad de Viña del Mar, Chile. 


\section{MATERIAL Y MÉTODO}

Estudio de tipo cuantitativo transversal, no experimental. Los investigadores diseñaron un instrumento de medición tipo cuestionario con 12 aseveraciones sobre $\mathrm{Cl}$ (siete sobre generalidades y cinco sobre excepciones a su aplicación) con tres posibles opciones de respuesta (correcto/incorrecto/no sabe) (Fig. 1). Éste fue modificado y validado por tres expertos.

El universo muestral informado por la Directiva de la Clínica Institucional fue:

- estudiantes de cuarto y quinto año de la Carrera de Odontología: 271.

- docentes de ramos clínicos: 58.

- pacientes ingresados durante el año 2019 a la Clínica a la fecha del estudio: 260
Previo a la aplicación del instrumento de medición, a cada sujeto se le proporcionó un $\mathrm{Cl}$ diseñado especialmente para esta investigación, cuyo protocolo se encuentra aprobado por el Comité de Ética Científica institucional (Acta CEC n²1/2018 y n01/2019).

A cada sujeto de estudio que consintió participar en la investigación, se le dio 10 minutos para contestar el cuestionario. A cada respuesta correcta se le asignó un punto. Quienes obtuvieron entre 9-12 puntos, se les consideró con un conocimiento alto; entre 5-8, regular; y de 0-4, bajo.

Para el análisis de los datos obtenidos, se utilizó el test chi-cuadrado. Los análisis estadísticos y gráficos fueron desarrollados en el software estadístico Stata 11.2.

\section{CUESTIONARIO: Conocimiento sobre el consentimiento informado en relación a la}

LEY N`24.584 "Deberes y Derechos de los pacientes"

\begin{tabular}{|c|c|c|c|}
\hline EDAD: & SEXO: & & \\
\hline \multicolumn{4}{|l|}{ Marque si Ud. es: } \\
\hline PACIENTE: & DOCENTE: & ALUMNO: IV & V \\
\hline
\end{tabular}

Marque con una " $\mathrm{X}$ " si considera correcta, incorrecta o no sabe, el contenido de cada aseveración:

\begin{tabular}{|c|c|c|c|}
\hline Aseveraciones & Correcto & Incorrecto & No sé \\
\hline $\begin{array}{l}\text { 1.- El consentimiento informado consiste en información adecuada, } \\
\text { oportuna y comprensible, por parte del profesional de salud, acerca del } \\
\text { diagnóstico, tratamiento y riesgos de una patología. }\end{array}$ & & & \\
\hline $\begin{array}{l}\text { 2.- El consentimiento informado debe ser entregado de forma escrita } \\
\text { siempre. }\end{array}$ & & & \\
\hline $\begin{array}{l}\text { 3.- El Consentimiento Informado puede ser firmado únicamente por el } \\
\text { paciente afectado. }\end{array}$ & & & \\
\hline $\begin{array}{l}\text { 4.- La aplicación del Consentimiento informado es obligatoria solo por } \\
\text { parte de los prestadores de salud pública. }\end{array}$ & & & \\
\hline $\begin{array}{l}\text { 5.- El profesional de la salud tiene la obligación de entregar } \\
\text { información sobre alternativas del tratamiento y valores asociados a } \\
\text { estos. }\end{array}$ & & & \\
\hline $\begin{array}{l}\text { 6.- Cuando el paciente no se encuentra capacitado para recibir } \\
\text { información, padezca dificultades de entendimiento o se encuentre } \\
\text { inconsciente, la información se le entrega al representante legal. }\end{array}$ & & & \\
\hline $\begin{array}{l}\text { 7.- En atenciones de emergencia o urgencia, el paciente se encuentra } \\
\text { sin acompañante, y este no puede entregar el consentimiento para la } \\
\text { atención, solo se realizan maniobras paliativas hasta contactar algún } \\
\text { familiar o que el paciente se encuentre con las facultades de decidir su } \\
\text { tratamiento. }\end{array}$ & & & \\
\hline $\begin{array}{l}\text { 8.- En situaciones que ponen en riesgo la vida del paciente, se puede } \\
\text { omitir el consentimiento informado. }\end{array}$ & & & \\
\hline $\begin{array}{l}\text { 9.- Los prestadores deberán adoptar las medidas necesarias para } \\
\text { asegurar la adecuada confidencialidad durante la entrega de esta } \\
\text { información, así como la existencia de lugares apropiados para ello. }\end{array}$ & & & \\
\hline $\begin{array}{l}\text { 10.- Toda persona tiene derecho a otorgar o denegar su voluntad para } \\
\text { someterse a cualquier procedimiento o tratamiento vinculado a su } \\
\text { atención de salud. }\end{array}$ & & & \\
\hline $\begin{array}{l}\text { 11.- El proceso se efectuará en forma verbal, pero deberá constar con } \\
\text { respaldo escrito, en caso de cirugías o procedimientos invasivo. }\end{array}$ & & & \\
\hline 12.- El paciente autoriza únicamente con su firma en un documento. & & & \\
\hline
\end{tabular}

Fig. 1. Cuestionario "Conocimiento sobre Consentimiento Informado". 


\section{RESULTADOS}

La encuesta fue respondida por un total de 389 personas: 130 estudiantes (33,42\%), 33 docentes $(8,48 \%)$ y 260 pacientes $(58,10 \%)$. Del total de participantes, un $61,92 \%$ correspondió a mujeres.

Respecto al conocimiento de los estudiantes, encontramos que, en su mayoría, presentan un conocimiento alto sobre $\mathrm{Cl}$, no encontrándose diferencia estadísticamente significativa entre los de cuarto y quinto año de la Carrera (valor-p 0,3041) (Tabla I).

Un $60,61 \%$ de los docentes muestran un grado de conocimiento regular, mientras que un $39,39 \%$ presentan un conocimiento alto. Ninguno presentó conocimiento bajo. Los pacientes, en su mayoría, presentan un conocimiento regular $(58,41 \%)$ (Tabla I).

Al realizar el análisis de proporciones, se obtuvo que entre los tres grupos el valor-p fue 0,004 , por lo tanto, existe diferencia significativa con relación al grado de conocimiento alto. Al comparar la dupla docente-estudiante el valor-p fue de 0,0084, por tanto, el conocimiento obtenido en los estudiantes es significativamente mayor que el de los docentes. Por otra parte, al comparar el resultado entre la dupla docentes-pacientes el valor-p fue 0,2580 , por lo tanto, no existe diferencia estadísticamente significativa en el conocimiento sobre $\mathrm{Cl}$ de ambos grupos. Por último, al comparar entre estudiantes-pacientes el resul- tado fue valor-p 0,0000, por lo tanto, se puede concluir que los estudiantes tienen un grado de conocimiento significativamente mayor.

Al redistribuir los sujetos de estudio según edad (informada en 358 casos), encontramos que, al realizar la prueba de proporciones para grado de conocimiento alto para tres grupos etarios, se obtuvo un valor-p 0,683, es decir, no hay diferencia significativa entre los grupos. Lo mismo ocurre al evaluar los resultados para conocimiento regular. Mientras que al evaluar el grado de conocimiento alto entre sexo hombre/mujer, se obtuvo un valor-p 0,0642, por lo tanto, se concluye que no existe diferencia estadísticamente significativa en el grado de conocimiento entre ambos sexos (Tabla II).

Finalmente, se realizó un análisis de los resultados obtenidos por cada pregunta. La primera afirmación, que dice relación con la definición de $\mathrm{Cl}$, fue la que obtuvo un mayor porcentaje de respuestas correctas $(83,85 \%)$. De las 5 afirmaciones relacionadas con excepciones a la firma del $\mathrm{Cl}$, una de ellas fue la que obtuvo el menor porcentaje de aciertos ("El Cl puede ser firmado únicamente por el paciente afectado"), con un 37,96 \%; mientras que dos de ellas obtuvieron la mayor cantidad de respuestas "no sabe" (aplicación de $\mathrm{Cl}$ en situaciones de emergencia vital y en presencia de paciente sin posibilidad de consentir), con $17,7 \%$ cada una.

Tabla I. Distribución de los encuestados según grupo de estudio y grado de conocimiento.

\begin{tabular}{|c|c|c|c|c|c|c|c|}
\hline \multirow[t]{2}{*}{ Encuestado } & \multicolumn{2}{|l|}{ Bajo } & \multicolumn{2}{|c|}{ Regular } & \multicolumn{2}{|l|}{ Alto } & \multirow{2}{*}{$\begin{array}{l}\text { Total } \\
\mathrm{N}\end{array}$} \\
\hline & $\mathrm{N}$ & $\%$ & $\mathrm{~N}$ & $\%$ & $\mathrm{~N}$ & $\%$ & \\
\hline Docente & 0 & 0,00 & 20 & 60,61 & 13 & 39,39 & 33 \\
\hline Estudiante $4^{\circ}$ Año & 1 & 1,30 & 29 & 37,66 & 47 & 61,04 & 77 \\
\hline Estudiante $5^{\circ}$ Año & 0 & 0,00 & 16 & 30,19 & 37 & 69,81 & 53 \\
\hline Paciente & 27 & 11,95 & 132 & 58,41 & 67 & 29,65 & 226 \\
\hline Total & 28 & 7,46 & 197 & 50,64 & 164 & 41,90 & 389 \\
\hline
\end{tabular}

Tabla II. Distribución de los encuestados según edad, sexo y grado de conocimiento.

\begin{tabular}{lccccccc}
\hline Encuestado & \multicolumn{2}{c}{ Bajo } & \multicolumn{2}{c}{ Regular } & \multicolumn{2}{c}{ Alto } & \multicolumn{2}{c}{ Total } \\
\cline { 2 - 8 } & $\mathrm{N}$ & $\%$ & $\mathrm{~N}$ & $\%$ & $\mathrm{~N}$ & $\%$ & $\mathrm{~N}$ \\
\hline Entre 18 y 25 Años & 4 & 4,88 & 46 & 56,10 & 32 & 39,02 & 82 \\
Entre 26 y 50 Años & 18 & 8,37 & 104 & 48,37 & 93 & 43,26 & 215 \\
Mayor a 50 Años & 5 & 8,20 & 32 & 52,46 & 24 & 39,34 & 61 \\
Total & 27 & 7,54 & 182 & 50,84 & 149 & 41,62 & 358 \\
\hline Mujer & 17 & 7,08 & 116 & 48,33 & 107 & 44,58 & 240 \\
Hombre & 13 & 8,16 & 81 & 55,10 & 55 & 36,73 & 149 \\
Total & 30 & 7,49 & 197 & 50,90 & 162 & 41,60 & 389 \\
\hline
\end{tabular}




\section{DISCUSIÓN}

Este es el primer estudio chileno que mide grado de conocimiento sobre el proceso de $\mathrm{Cl}$ en grupos de estudiantes de pregrado, docentes y pacientes de manera conjunta desde la implementación de la Ley 20.584. En este estudio se constató que el grupo con mayor conocimiento sobre $\mathrm{Cl}$ fue el de estudiantes, sin distinción del año que cursaban, seguido por el de los docentes y pacientes.

En España, desde el año 2002, existe la Ley de Autonomía del Paciente (LAP), la que se puede considerar homóloga en sus contenidos con la Ley de Derechos y Deberes del Paciente chilena. Un estudio realizado durante el año 2008 en dos hospitales universitarios de Sevilla midió el conocimiento que presentaban profesionales sanitarios (médicos y auxiliares de enfermería) respecto a esta ley, demostrándose el desconocimiento del personal sanitario acerca de aspectos claves de ella. Esto fue consistente con la poca divulgación de la misma y la poca iniciativa de las personas por conocerla, pero contradictorio con la importancia que se le otorga en el desempeño del trabajo (Fernández Garrido et al., 2009). Más adelante, en 2012, un estudio realizado en un Hospital Universitario de Granada, evaluó el conocimiento sobre la LAP que tenía un grupo de médicos y enfermeros mediante sus respuestas a un cuestionario anónimo. Los resultados mostraron que los profesionales encuestados tenían buena formación respecto al derecho de los pacientes a recibir información, aunque algunos de ellos desconocían aspectos fundamentales de su deber legal (Arenas López et al., 2012). En nuestro estudio, encontramos que un porcentaje importante de los profesionales docentes clínicos $(60,61 \%)$ tenía sólo un conocimiento regular sobre $\mathrm{Cl}$, mientras que un porcentaje menor $(39,39$ $\%)$, tenía un conocimiento alto. Esto podría explicarse dada la data relativamente reciente de la Ley, considerando que no todos quienes se desempeñan como docentes tuvieron dentro de sus contenidos de pregrado tópicos relacionados con Cl. Es importante aumentar la investigación del tema en el área odontológica, de modo de poder cumplir con la aplicación del $\mathrm{Cl}$ de manera correcta, considerando las sutilezas de la atención odontológica: cortos tiempos de atención en el sistema público de salud, procedimientos, en su mayoría, invasivos, atenciones relacionadas a dolor agudo, riesgo de infecciones, etc. Sumado a esto, y en concordancia con los autores de los estudios antes mencionados, consideramos que es también fundamental la formación continua que introduzca conocimientos sobre derecho sanitario, $\mathrm{Cl}$ y autonomía del paciente. En Chile, a la luz de los resultados de nuestro estudio, creemos importante reforzar los tópicos referidos a excepciones a la aplicación de $\mathrm{Cl}$ : qué hacer si el paciente es menor de edad, está inconsciente o es considerado incapaz, o bien, si nos encontramos ante una situación de urgencia vital o cuando el paciente se niega a firmarlo. Todo lo anterior en pos de concientizar a los profesionales de salud, cumplir con el mandato legal y contribuir al ejercicio pleno del derecho a la autonomía de nuestros pacientes.

Respecto al conocimiento de los estudiantes de pregrado, en un Hospital de Durango en México se aplicó un cuestionario para medir el conocimiento sobre $\mathrm{Cl}$ que tenían pasantes de medicina, enfermería y odontología. Cabe considerar que, en ese país, desde 1986, el deber legal de la aplicación de Cl está regulado en la Ley General de Salud en Materia de Prestaciones de Servicios de Atención Médica. Los resultados de ese estudio mostraron que los participantes presentan importantes deficiencias sobre el conocimiento que tienen de $\mathrm{Cl}$, obteniendo una calificación promedio deficiente y que se hace necesario fortalecer los conocimientos de los estudiantes en esta área (Galván Meléndez et al., 2016). En el presente estudio, encontramos que los estudiantes de cuarto y quinto año de la carrera presentan un alto conocimiento sobre el proceso de $\mathrm{Cl}$, significativamente mayor al conocimiento demostrado por los pacientes e, incluso, por sus propios docentes. Esta llamativa situación podría explicarse ya que todos ellos se han formado posterior a la entrada en vigencia de la Ley $20.584 \mathrm{y}$, por tanto, han incorporado este paradigma de horizontalidad en la relación médico-paciente y respeto por la autonomía en su modo de entender la profesión sanitaria, además de un mayor acceso a la información propio de los tiempos que corren.

Los pacientes, en su mayoría, presentan un conocimiento regular sobre $\mathrm{Cl}$, seguido por quienes tienen un conocimiento alto y por un $11,95 \%$ de quienes tienen uno bajo. Cabe destacar que, desde la entrada en vigencia de la Ley 20.584 , se exige que en todos los lugares de alta concurrencia de público de los servicios de salud (hall de ingreso, sala de espera, oficina de informaciones, etc.), tanto públicos como privados, se encuentre exhibida la "Carta de Derechos y Deberes de los Pacientes" (Fig. 2), en donde se representan de manera gráfica y escrita los aspectos más relevantes señalados en la Ley. 


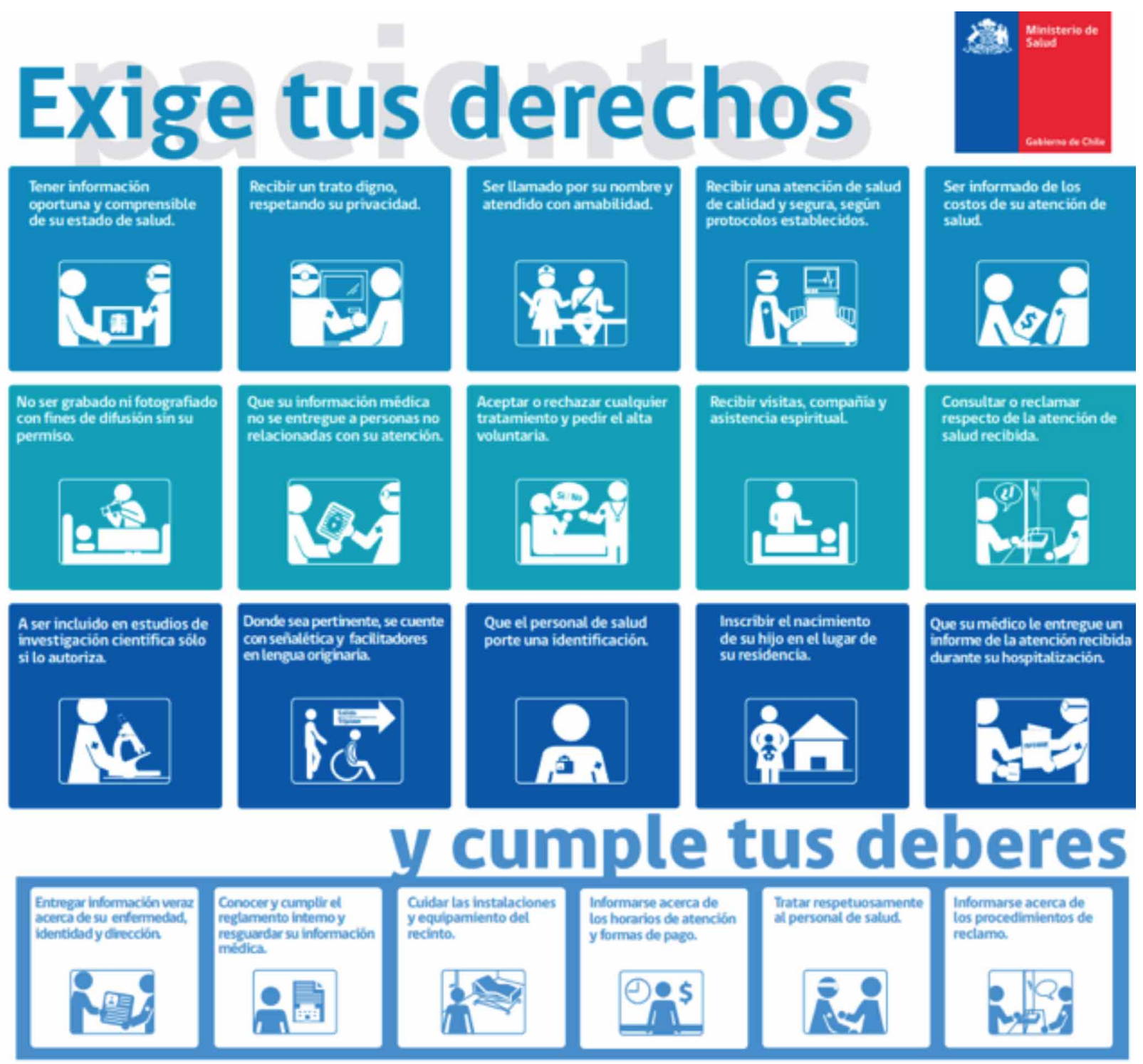

TODA PERSONA PODRA RECLAMAR SUS DERECHOS ANTE EL CONSULTORIO, HOSPITAL, CLINICA O CENTRO MEDICO PRIVADO QUE LO ATIENDE. SI LA RESPUESTA NO ES SATISFACTORIA PODRA RECURRIR A LA SUPERINTENDENCIA DE SALUD. (Extracto de la Ley N²0.584 de Derechos y Deberes de los Pacientes).

Fig. 2. Carta de Derechos y Deberes del Paciente en Relación a la Atención de Salud

Si bien esta Carta incluye quince derechos y seis deberes del paciente, el proceso de $\mathrm{Cl}$ no se encuentra explícito dentro de ella y sólo puede desprenderse su uso del cuadrante que hace alusión al derecho "aceptar o rechazar cualquier tratamiento" (Ministerio de Salud, 2012b). El cerca de $12 \%$ de pacientes que presenta un conocimiento bajo sobre este importante procedimiento en salud nos hace plantearnos las siguientes preguntas: ¿tiene la Carta información suficiente? ¿es esta información graficada de manera lo suficientemente atractiva para llamar la atención de nuestros usuarios? ¿están los usuarios realmente interesados en saber más sobre el $\mathrm{Cl}$ ? ¿Hay una real comprensión de la información entregada? El alcance del presente estudio no permite responder a todas estas interrogantes, pero evidencia una realidad de la cual como tratantes debemos hacernos cargo: somos los llamados a educar y empoderar a nuestros pacientes en los aspectos relacionados a su salud. Para este fin, debemos procurar estar en constante actualización y transmitir los conocimientos a los futuros profesionales. De otra manera, estaremos privando a nuestros usuarios del ejercicio pleno de su autonomía. 
LOZA, R. C.; SALGADO, M. B.; SÁNCHEZ, V. J.; SARMIENTO, I. F. \& LAGOS, T. D. Knowledge on informed consent in teachers, students and patients of the faculty of dentistry. Int. J. Odontostomat., 14(3):424-429, 2020.

ABSTRACT: The objective of this study was to determine the level of knowledge regarding the Informed Consent procedure presented in a sample of teachers, students and dental patients. An anonymous questionnaire was applied to 271 students, 58 teachers and 260 patients of a Teaching Dental Clinic. The data analysis was performed by applying the chi-square test. The majority of the students have a high knowledge. $60.61 \%$ of the teachers show a level of regular knowledge and $39.39 \%$ show a high knowledge. Most of the patients show a regular knowledge $(58.41 \%)$. It is important to increase both the research of the subject and the continuous training that introduces knowledge about patient autonomy. In this way, we will raise awareness among teachers in the area and strengthen the knowledge of new generations, contributing our legal mandate and the full exercise of the right to autonomy of our patients.

KEY WORDS: informed consent, patient autonomy, patient rights.

\section{REFERENCIAS BIBLIOGRÁFICAS}

Arenas López, A.; Castellano Arroyo, M.; Miranda León, M. T. \& Reche Molina, A. Conocimiento y cumplimiento de los profesionales sanitarios del derecho del paciente a la información clínica. Rev. Esp. Med. Legal, 38(1):11-6, 2012.

Centro Interdisciplinario de Estudios en Bioética (CIEB). Ética y Odontología. Una Introducción. Santiago de Chile, Centro Interdisciplinario de Estudios en Bioética (CIEB), Vicerrectoría de Investigación y Desarrollo, Universidad de Chile, Escritos de Bioética $\mathrm{N}^{\circ} 2,2006$

Fernández Garrido, C.; Lopera Uribe, G. E.; Méndez Pérez, L.; Otero Espiga, A.; Gallardo Abril, M. G. \& Lagoa Elías, C. Conocimiento de la ley de autonomía del paciente por el personal médico y de enfermería implicado en el proceso quirúrgico. Enferm. Clin., 19(6):330-4, 2009.

Galván Meléndez, M. F.; Huerta Guerrero, H. M.; Galindo Burciaga, M.; Barrientos Ramos, A. A. \& Morales Castro, M. E. Conocimiento del consentimiento informado clínico en profesionales en formación en salud. Investig. Edud. Med., 5(18):108-14, 2016.

Ministerio de Salud. Decreto 570. Aprueba Reglamento para la Internación de las Personas con Enfermedades Mentales y sobre los Establecimientos que la Proporcionan. Santiago de Chile, Ministerio de Salud, Gobierno de Chile, Diario Oficial de la República de Chile, 2000.

Ministerio de Salud. Ley n'19.451. Establece Normas Sobre Trasplante y Donación de Órganos. Diario Oficial de la República de Chile. Santiago de Chile, Ministerio de Salud, Gobierno de Chile, 2019.

Ministerio de Salud. Ley No19.779. Establece Normas Relativas al Virus de Inmunodeficiencia Humana y crea Bonificación Fiscal para Enfermedades Catastróficas. Diario Oficial de la República de Chile. Santiago de Chile, Ministerio de Salud, Gobierno de Chile, 2017.
Ministerio de Salud. Manual Técnico que Contiene la Normativa gráfica que Establece el Correcto uso de la Carta de Derechos y Deberes de los Pacientes en las Instituciones de Salud. Santiago de Chile, Ministerio de Salud, Gobierno de Chile, 2012a.

Ministerio de Salud. Resolución Exenta $N^{\circ} 605$. Determina Contenido de la Carta de Derechos y Deberes del Paciente en Relación a la Atención de Salud. Diario Oficial de la República de Chile. Santiago de Chile, Ministerio de Salud, Gobierno de Chile, 2012b.

Subsecretaría de Salud Pública. Ley N²0.584. Regula los Derechos y Deberes que tienen las Personas en Relación con Acciones Vinculadas a su Atención en Salud. Diario Oficial de la República de Chile. Santiago de Chile, Subsecretaría de Salud Pública, Ministerio de Salud, Gobierno de Chile, 2019.

Dirección para correspondencia:

Denisse Lagos Tissie

Escuela de Ciencias de la Salud

Carrera de Odontología

Universidad Viña del Mar

Viña del Mar

CHILE

Email: Lt.denisse@gmail.com

Recibido : 30-01-2020

Aceptado: 17-04-2020 